\title{
An unusual case of apical hypertrophic cardiomyopathy in a transplanted heart
}

\section{Un caso non usuale di cardiomiopatia ipertrofica apicale in un cuore trapiantato}

\author{
Antonello D'Andrea, Raffaella Scarafile, llaria Ferrara, Maria Luisa De Rimini1, \\ Pietro Muto', Maria Giovanna Russo, Raffaele Calabrò
}

Keywords: cardiac transplantation; contrast echocardiography; apical hypertrophic cardiomyopathy; cardiac magnetic resonance.

Monaldi Arch Chest Dis 2010; 74: 192-194.

Chair of Cardiology - Second University of Naples - Monaldi Hospital Naples, Italy.

1 Department of Nuclear Medicine - Monaldi Hospital - Naples, Italy.

Corresponding author: Antonello D'Andrea, MD - Via G. Martucci 35, 80121 - Naples, Italy; Tel.: ++390817618525, Fax: ++390817145205, E-mail:antonellodandrea@libero.it

\section{Case Report}

A 66-year-old man who had undergone cardiac transplantation 20 years ago (in 1990) for idiopathic dilated cardiomyopathy was referred for the first time to our hospital because of palpitations and dyspnoea during moderate physical effort (especially during the last 6 months).

At the admission, the patient exhibited a previous electrocardiogram (ECG) and echocardiogram, both performed in 1994. The ECG showed left ventricular (LV) hypertrophy and negative $\mathrm{T}$ waves on the precordial leads, the echocardiogram showed only mild LV hypertrophy, with poor LV apical endocardial definition. The patient also showed a coronary angiography performed the same year, with coronary arteries and ventriculography within normal limits. In addition, routine cardiac biopsy results were normal until 1 year after the transplantation.

Physical findings were within normal limits. The ECG showed negative $\mathrm{T}$ waves on the precordial leads (Figure 1). Standard echocardiography showed normal LV ejection fraction and LV hypertrophy mainly located in the LV apex (Figure $2 \mathrm{~A}$ ). Colour Doppler highlighted systolic aliasing at the level of the distal third and of the apical portions of the left ventricle (Figure 2 B). By pulsed-wave Doppler, the gradient between the apex and base of the left ventricle was higher than $30 \mathrm{mmHg}$. By 3D echocardiography, a maximal apical LV wall thickness of $20 \mathrm{~mm}$ was measured at end-diastole in the apical 4-chamber view (Figure $2 \mathrm{C}$ ). By use of intravenous contrast echocardiography, the typical "ace of spades" configuration, the hallmark diagnostic criteria for apical hypertrophic cardiomyopathy (HCM), was clearly evidenced (Figure $2 \mathrm{D}$ ). For further tissue characterization, late gadolinium enhancement (LGE) cardiovascular magnetic reso- nance (CMR) was performed. Steady-state free precession images and inversion recovery LGE images in the long- and short-axis images showed sub-endocardial enhancement in the hypertrophied LV apex, in absence of regional wall-motion abnormalities (Figure 2 E-F). Our patient was treated with oral Beta-blocker and Ace-inhibitor after diagnosis, and symptoms improved after drug therapy.

To the best of our knowledge, this is the first case of an apical HCM developing in another body of a cardiac transplant recipient patient. Even if echocardiography is the first line imaging modality for the followup of cardiac transplant patients, it is well known that the cardiac apex may be difficult to visualize since postoperative echocardiography windows are often of poor quality because of intervening air spaces around the heart. In some cases, apical hypertrophy may be confused with apical thrombus. Echocardiographic studies with intravenous contrast agents suggest that, in the majority of these cases, poor apical endocardial definition leads to false negative reports. Other noninvasive modalities such as stress perfusion scintigraphy may suggest an enhanced tracer uptake in the apical area of the left ventricle. The definite diagnosis of apical HCM with spade-like configuration was based on published criteria of both an apical wall thickness of $15 \mathrm{~mm}$ and a ratio $>1.3$ of maximum left ventricular short axis thickness at the apical level to the basal level [1-3]. CMR can image in any plane and the introduction of steady state free precession sequences has resulted in a substantial improvement in blood to myocardium contrast, which is ideal for consistent and accurate imaging of the apex [4-5].

Cardiac catheterization is frequently done in these patients due to similarity in symptoms and ECG findings with acute coronary syndromes. Ventriculography shows the classic sign of systolic obliteration of the apex and a small ventricular cavity at the base. 


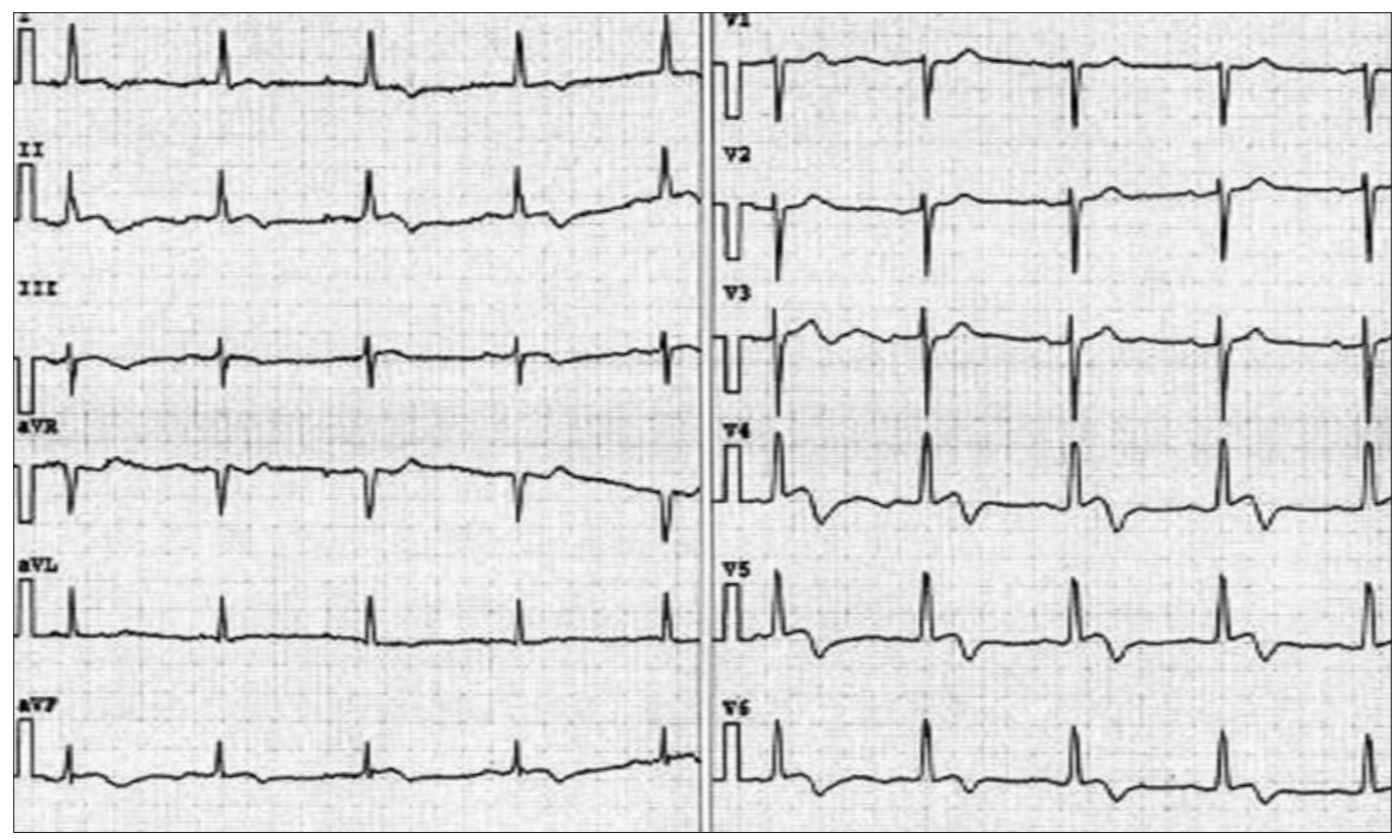

Figure 1. - The more recent electrocardiogram confirmed negative $\mathrm{T}$ waves on the precordial leads.

Of note, in our patient it would have been clinically relevant to perform a genetic analysis using previous myocardial biopsy results. However, since the patient was referred for the first time to our hos- pital in 2010, the myocardial samples obtained after cardiac transplantation were not available.

In conclusion, western patients with apical HCM are thought to require the same risk stratification and

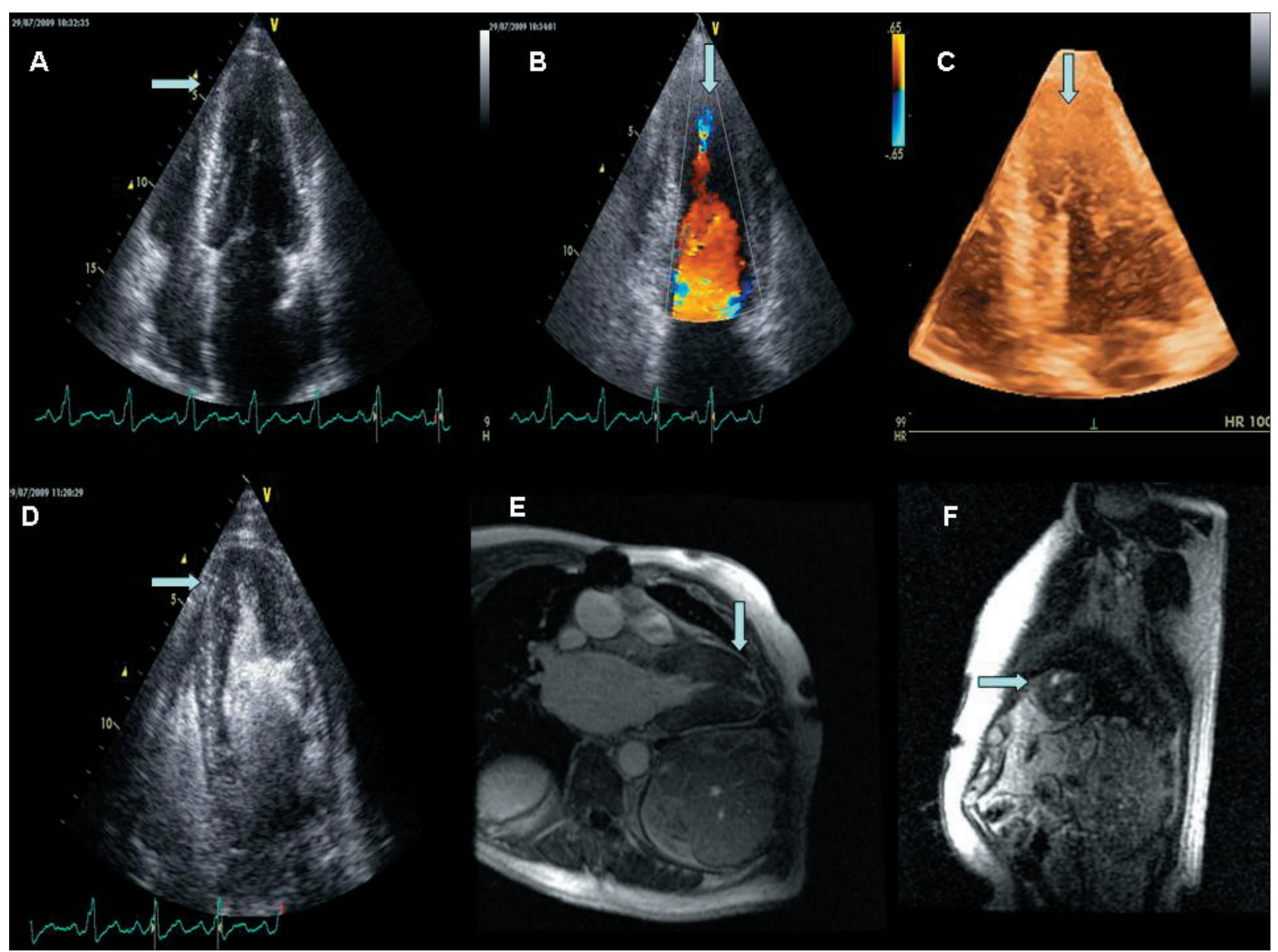

Figure 2. - Panel A: standard echocardiography showing normal LV ejection fraction and LV hypertrophy mainly located in the LV apex. Panel B: Colour Doppler echocardiography showing systolic aliasing at the level of the apical portions of the left ventricle (arrow)

Panel C: 3D echocardiography. Maximal apical LV wall thickness of $20 \mathrm{~mm}$ was measured at end-diastole in the apical 4-chamber view (arrow). Panel D: intravenous contrast echocardiography. The typical "ace of spades" configuration was clearly evidenced.

Panel E-F: Cardiac Magnetic Resonance. Inversion recovery late-gadolinium images in the long- (Panel E) and short-axis (Panel F) images showed sub-endocardial enhancement in the hypertrophied LV apex (arrow). 
family screening as other HCM patients and the implications of a missed diagnosis are significant. In fact, although most of patients with apical HCM have a benign course, occasionally these patients develop arrhythmias requiring treatment with antiarrhythmic drugs or implantable defibrillator device.

Therefore, we believe that a complete ultrasound investigation, including intravenous contrast echocardiography and/or stress echocardiography, should be performed in cardiac transplant donors and recipients when the ECG raises suspicion of apical HCM and/or standard echocardiographic results are inconclusive.

\section{References}

1. Spencer KT, Bednarz J, Mor-Avi V, Weinert L, Tan J, Godoy I, et al. The role of echocardiographic harmonic imaging and contrast enhancement for improvement of endocardial border delineation. $J$ Am Soc Echocardiogr 2000; 13: 131-8.

2. Ward RP, Weinert L, Spencer KT, et al. Quantitative diagnosis of apical cardiomyopathy using contrast echocardiography. J Am Soc Echocardiogr 2002; 15: 316-22.

3. D'Andrea A, Liccardo B, Scarafile R, Esposito N, Calabrò R. Apical hypertrophic cardiomyopathy: quickly noninvasive diagnosis by intravenous contrast echocardiography. Minerva Cardioangiol 2008; 56 (5): 578-9.

4. Suzuki J, Shimamoto R, Nishikawa J, et al. Morphological onset and early diagnosis in apical hypertrophic cardiomyopathy: a long term analysis with nuclear magnetic resonance imaging. J Am Coll Cardiol 1999; 33: 146-51.

5. Moon JC, Fisher NG, McKenna WJ, et al. Detection of apical hypertrophic cardiomyopathy by cardiovascular magnetic resonance in patients with non-diagnostic echocardiography. Heart 2004; 90: 645-649. 